\title{
EL BLOG COMO HERRAMIENTA DE SOPORTE SOCIAL PARA PERSONAS CON ENFERMEDAD CRÓNICA ${ }^{1}$
}

\author{
A BLOG AS A SOCIAL SUPPORT TOOL FOR PEOPLE \\ WITH CHRONIC DISEASE
}

\author{
Gloria Mabel Carrillo * \\ Lorena Chaparro Díaz ${ }^{* *}$ \\ LUCY BARRERA ORTIZ ${ }^{* * *}$ \\ Natividad Pinto Afanador ${ }^{* * *}$ \\ BEATriz SÁNChEZ HERRERA ${ }^{* * * *}$
}

\begin{abstract}
RESUMEN
Objetivo: Examinar el fenómeno del soporte social con el uso del blog paratucuidadoenlinea en personas con enfermedad crónica. Método: Investigación descriptiva exploratoria con abordaje cualitativo, realizada en Bogotá en el 2010, a través de un estudio de casos con 8 personas con enfermedad crónica que se vincularon al blog paratucuidadoenlinea. La intervención tuvo una duración de cuatro meses y consistió en brindar un servicio de 16 horas diarias disponibles en el blog para atender a los casos. La información se obtuvo mediante observaciones a través de diarios de campo de cada uno de los casos y entrevistas semiestructuradas realizadas al finalizar la intervención. El análisis de la información incluyó la organización de los informes de diarios de campo de cada caso, transcripción de las participaciones de los casos a través del chat y/o foros y las entrevistas realizadas, construcción de códigos y categorías que describen la percepción de las personas respecto a la estrategia. Resultados: El blog fue utilizado para obtener soporte social, indistintamente del nivel socioeconómico, edad o grado de escolaridad, de los casos. Las categorías resultantes fueron: autoreconocimiento y crecimiento personal, vínculo, comunicación, utilidad, satisfacción y respuestas. Conclusiones. Se requiere explorar las estrategias utilizadas a través del blog, incluir redes de soporte social en línea y generar una escala de soporte social percibido con uso de tecnologías, acorde al contexto latinoamericano.
\end{abstract}

Palabras clave: Apoyo social, enfermedad crónica, sistemas de apoyo a decisiones clínicas, desarrollo tecnológico.

\begin{abstract}
To examine the phenomenon of social support using the blog paratucuidadoenlinea in people with chronic illness. Method: Exploratory descriptive research with a qualitative approach, held in Bogotá in 2010, through a case study with 8 people with chronic illness who were linked to paratucuidadoenlinea blog. The intervention lasted four months and consisted of providing a service available 16 hours a day on the blog to respond to cases. The information was obtained by observations in field journals of each of the cases and semi-structured interviews conducted after the intervention. The data analysis included the

\footnotetext{
${ }^{1}$ Grupo de Cuidado al Paciente crónico y la familia, Facultad de Enfermería, Universidad Nacional de Colombia y Red Latinoamericana de Cuidado al Paciente Crónico.

*Enfermera, Profesora Facultad de Enfermería, Universidad Nacional de Colombia. Colombia. Email: gmcarrillog@unal.edu.co

** Enfermera, Profesora Facultad de Enfermería, Universidad Nacional de Colombia. Colombia. Email: olchaparrod@unal.edu.co

${ }^{* * *}$ Enfermera, Profesora Facultad de Enfermería, Universidad Nacional de Colombia. Colombia. Email: 1barrerao@unal.edu.co

${ }^{* * * *}$ Enfermera, Profesora Facultad de Enfermería, Universidad Nacional de Colombia. Colombia. Email: rnpintoa@unal.edu.co

${ }^{* * * * *}$ Enfermera, Profesora Facultad de Enfermería, Universidad Nacional de Colombia. Colombia. Email: cbsanchez@unal.edu.co
} 
organization of daily reports from the field in each case, transcription of the holdings of the cases through the chat and/or forums and interviews, building codes and categories that describe the perception of individuals with regard to the strategy. Results: The blog was used to obtain social support, regardless of socioeconomic status, age or education level, of the cases. The resulting categories were: self-recognition and personal growth, connection, communication, utility, satisfaction and responses. Conclusions: Is required to explore strategies used throughout the blog, include social support networks online and generate a scale of perceived social support use of technology, according to the Latin American context.

Key words: Social support, chronic disease, decision support systems clinical, technological development.

Fecha recepción: 25/07/11 Fecha aceptación: 25/11/11

\section{INTRODUCCIÓN}

Son múltiples las necesidades de cuidado a largo plazo y de manera continua que requieren las personas con enfermedades crónicas y sus cuidadores familiares (1), con responsabilidades directas sobre el equipo de salud y generadas para escenarios extra e intrahospitalarios. El soporte social, se considera un recurso clave para abordar estas necesidades de cuidado, al cumplir un papel determinante como efecto mediador dentro de las experiencias vitales estresantes y la enfermedad, reducir significativamente el impacto percibido por esta, favorecer mayor adaptación a las situaciones, con repercusiones directas en la calidad de vida de las personas que padecen las dolencias crónicas (2).

Además, se ha identificado que el soporte social favorece la continuidad de la atención en salud, haciendo parte de las estrategias propuestas en las políticas mundiales de cuidado para las personas con enfermedades (1).

Las tecnologías de la información y la comunicación (TICs), se han descrito como herramientas para brindar el soporte social, se definen como "estrategias para conformar redes de apoyo y proporcionar un sistema de soporte con mayor seguimiento, en el que se integran conexiones través de medios de comunicación y soporte en línea, facilitando interacciones permanentes entre las personas con enfermedad crónica, sus cuidadores familiares y el sistema de salud" (1, 3-6).

Las TICs permiten que se recoja, sistematice y difunda información para fomentar el desarrollo de conocimientos y habilidades en las personas con enfermedades crónicas y sus cuidadores $(7,8)$, facilitan procesos de adaptación frente a la situación con cambios respecto a comportamientos y estilos de vida $(9,10)$ en la medida en que se eliminan barreras de accesibilidad y altos costos de los servicios $(11,12)$.

El soporte social con TICs es establecido por medio de redes sociales con objetivos comunes, como una herramienta de integración donde se involucran sectores académicos, científicos, tecnológicos y culturales mediante un sistema de comunicación para apoyar procesos, compartir experiencias, fortalecer conocimientos y unir esfuerzos en la construcción permanente que incluye el desarrollo individual y lo colectivo $(12,13)$.

En este soporte se reconocen cuatro componentes fundamentales: 1) la red social, 2) la interacción social, 3 ) el soporte social percibido y 4) el soporte instrumental (7).

Dentro de los estudios que han reportado beneficios y ventajas significativas de brindar soporte social con el uso de TICs se destacan el de Grant et al. (14), quienes a través de una intervención telefónica dirigida a cuidadores familiares de personas sobrevivientes de accidente cerebrovascular, concluyen que los participantes lograron potencializar la vitalidad, la salud mental, las habilidades de resolución de problemas y la preparación 
en el cuidado y disminuyeron el negativismo, la impulsividad, la falta de autocuidado y la depresión relacionada con la carga del rol del cuidador.

Klemm y Wheeler (10), concluyen que los cuidadores de personas con cáncer, encuentran esperanza y empoderamiento al participar en grupos de apoyo en línea. Afirman que este tipo de soporte en línea es un factor protector contra el desgaste del cuidador.

Shaw et al. (15), en un estudio exploratorio de factores predictivos de participación en un equipo de apoyo en línea a un grupo de mujeres con cáncer de mama, refieren que la preocupación por la enfermedad, el impacto emocional por el diagnóstico, los tratamientos y sus efectos secundarios; así como los temores relacionados con la muerte, son variables que pueden condicionar la vinculación en los grupos de apoyo.

Matthew y Larose (16), evidenciaron que el apoyo en línea es relativamente sencillo y fácil de encontrar, que el internet es un medio social y puede ser utilizado para el intercambio de información de apoyo y que los espacios en línea (correo electrónico, chat, mensajería instantánea) permiten buscar apoyo y comunicarse con las personas que no se encuentran tradicionalmente en el círculo de relaciones sociales, al tiempo que se disminuye la ansiedad que representa estar frente a frente (cara a cara).

Barrera et al (2), en un estudio exploratorio, realizado en Bogotá en 2008 y 2009 con 144 cuidadores familiares de personas con enfermedad crónica describen los indicadores de estructura y proceso necesarios para el uso de las TICs y determinan su efectividad con la aplicación del instrumento "Inventario de soporte social en enfermedad crónica" (SSCI) (17), a partir de una intervención que consistió en el uso de videos y vinculación de los usuarios a una red de apoyo social.

Concluyen que esta intervención en su totalidad, es efectiva y positiva como soporte social para la mayoría de los cuidadores familiares. En el análisis por dimensiones, la de guía, información e instrucción y la de interacción personal, son las que reportan los efectos más altos, las de retroalimentación e interacción social obtienen los niveles más bajos.

Afirman que se requiere continuar en la identificación y diseño de TICs acordes al contexto de los usuarios, explorar herramientas como el internet y profundizar en las redes de soporte social en línea. A pesar de los beneficios y ventajas que reportan el uso de las TICs como estrategias para brindar soporte social, son pocas las experiencias documentadas en América Latina, y en Colombia que permitan evidenciar el grado de acceso, uso y apropiación, así como el soporte social percibido de las personas con enfermedades crónicas y cuidadores familiares, a partir de propuestas de intervención formales orientadas por el equipo de salud.

El objetivo del estudio fue Examinar el fenómeno del soporte social con el uso del blog paratucuidadoenlinea en personas con enfermedad crónica.

\section{MÉTODO}

Investigación exploratoria de tipo cualitativo, con la metodología de estudio de casos, para analizar el fenómeno de soporte social con el uso de un blog por parte de personas con enfermedad crónica. Este método fue elegido ya que permite determinar la dinámica de por qué y cómo la persona se comporta o desarrolla de forma particular dentro de una intervención propuesta de soporte social con uso de TICs.

Polit (18) afirma que la máxima prioridad de un estudio de casos es generar conocimientos sobre el caso específico, en ocasiones permitiendo que se puedan examinar fenómenos que no han sido estudiados con el suficiente rigor.

La investigación constó de tres etapas para su desarrollo: 
1. Diseño de una estrategia de soporte social en línea para personas con enfermedad crónica, que facilitara la interacción entre los participantes por medio de chat, foros y video foros, de acceso gratuito con la posibilidad de restringir la entrada de acuerdo a la determinación del grupo: Blog denominado paratucuidadoenlínea.

2. Implementación de la estrategia: Captación y vinculación de los participantes al blog, capacitación en el uso de la herramienta, montaje de un sistema de seguimiento para determinar indicadores de uso, acceso y apropiación de las TICs.

La intervención tuvo una duración de cuatro meses y consistió en brindar un servicio que estuvo 16 horas diarias disponibles en el blog para atender consulta y asesoría a las personas con enfermedad crónica, a través del chat y el foro. Así mismo para realizar el seguimiento de la participación por medio del contador de visitas.

La selección de los informantes se realizó de forma intencional teniendo en cuenta los siguientes criterios: Informantes decisores y motivadores de cambio al uso de herramientas de TICs y que participaran de las estrategias usadas para dar soporte social con tecnologías.

La información se obtuvo mediante observaciones a través de diarios de campo de cada uno de los casos y entrevistas semiestructuradas realizadas al finalizar la intervención.

Todas las intervenciones de los participantes a través del chat y/o el foro se transcribieron textualmente en los diarios de campo durante el periodo en que se mantuvo la intervención.

3. Análisis de la información: Este proceso se inició con la organización de los informes de diarios de campo de cada caso, la transcripción de las intervenciones a través del chat y/o el foro y las entrevistas realizadas. Posteriormente se extrajeron elementos que permitieron construir códigos con los que se definieron las categorías que describen la experiencia y percepción de los participantes respecto a la estrategia de intervención.

Es de resaltar que los códigos fueron elaborados con base en las expresiones de los informantes, utilizando el criterio de saturación teórica. Se utilizó el programa ATLAS.ti de investigación cualitativa para consolidar la información obtenida.

El estudio contempló los aspectos éticos referentes a consentimiento informado, participación voluntaria, el manejo confidencial de la información, y la autorización de las instancias pertinentes, así mismo contó con el aval de Comité de Ética de la Facultad de Enfermería de la Universidad Nacional de Colombia.

\section{RESULTADOS}

Se analizaron 8 casos de personas con enfermedades crónicas a las que se les dio acceso. A continuación se presenta un resumen de cada caso:

Caso 1: Mujer de 62 años, con diabetes tipo II, hipotiroidismo y un Cáncer de mama en tratamiento oncológico actualmente. Escolaridad: Bachillerato completo. Estado civil soltera. Ocupación actual: Hogar. Auto-calificación en habilidades en uso de computador: 2/10. Uso ocasional del internet, no cuenta con computador e internet en casa. Frecuenta salas de café internet y usa salas de informática de la Universidad Nacional de Colombia (UN). La capacitación que requirió para el uso de la estrategia fue de 12 horas. Muestra interés por la capacitación y el uso de la TIC. Al inicio de la intervención no tenía habilidades en el manejo del computador.

Caso 2: Mujer de 28 años, con una comunicación interauricular. Escolaridad: técnico. Estado civil: soltera. Ocupación: trabajo in- 
dependiente. Auto-calificación en habilidades en uso de computador: 8/10. Uso ocasional del internet. Cuenta con computador e internet en casa. No frecuenta salas de café internet ni usa salas de informática de la UN. La capacitación que requirió en el uso de la TIC fue de 5 horas. Es sometida a un tratamiento quirúrgico. Tiene conocimientos previos respecto al uso de la herramienta: internet.

Caso 3: Mujer de 64 años, con Cardiopatía coronaria. Escolaridad: técnico. Estado civil: soltera. Ocupación: Hogar. Auto-calificación en habilidades en uso de computador: 3/10. Uso ocasional del internet. No Cuenta con computador e internet en casa. Frecuenta salas de café internet y usa salas de informática de la UN. Horas de capacitación requeridas: 20. Presenta dificultades para utilizar la herramienta virtual, que implican mayor tiempo de capacitación por parte del orientador.

Caso 4: Mujer de 58 años, con Artritis Reumatoidea- hipoglucemia en estudio. Escolaridad: Bachillerato completo. Estado civil: soltera. Ocupación: Trabajo independiente. Auto-calificación en habilidades en uso de computador: 2/10. No usa el internet. No Cuenta con computador e internet en casa. No frecuenta salas de café internet. Usa salas de informática. Horas de capacitación requeridas: 10 y de forma virtual. Muestra interés por la capacitación y el uso de la TIC. Se evidencian dificultades para utilizar la herramienta virtual, referidos como dificultad de acceso y manejo del computador.

Caso 5: Hombre de 44 años, con Hipertensión Arterial y Cáncer de Pulmón. Escolaridad: Universidad completa. Estado civil: separado. Ocupación: Empleado. Auto-calificación en habilidades en uso de computador: 10/10. Usa el internet con mucha frecuencia. Cuenta con computador e internet en casa. No frecuenta salas de café internet ni usa salas de informática. Horas de capacitación requeridas: 2 y de forma virtual. Muestra interés por la capacitación y el uso de la TIC. Tiene conocimien- tos previos respecto al uso de la herramienta: internet.

Caso 6: Mujer de 48 años, con una Miocardiopatía. Escolaridad: Bachillerato incompleto. Estado civil: Unión libre. Ocupación: Hogar. Auto-calificación en habilidades en uso de computador: 8/10. Usa el internet ocasionalmente. Cuenta con computador e internet en casa. Frecuenta salas de café internet. No usa salas de informática. Horas de capacitación requeridas: 5 y de forma virtual. La persona muestra interés por la capacitación y el uso de la TIC.

Caso 7: Mujer de 18 años, con diagnóstico de Hipertensión Pulmonar- Hipertensión Arterial. Escolaridad: Bachillerato incompleto. Estado civil: Soltera. Ocupación: Estudiante. Auto-calificación en habilidades en uso de computador: 10/10 Usa el internet frecuentemente Cuenta con computador e internet en casa. No Frecuenta salas de café internet. No usa salas de informática. Horas de capacitación requeridas: 5 y de forma virtual.

Caso 8: Mujer de 27 años, con diagnóstico de Tromboembolismo pulmonar, Hipertensión arterial, Hipotiroidismo e hipoglicemia. Escolaridad: Universitaria completo. Estado civil: Casada. Ocupación: Empleada. Autocalificación en habilidades en uso de computador: 10/10. Usa el internet frecuentemente. Cuenta con computador e internet en casa. No Frecuenta salas de café internet. Horas de capacitación requeridas: $5 y$ de forma virtual. Muestra interés por la capacitación y el uso de la tic. La participante refiere agradecimiento por el acompañamiento.

\section{Capacitación y acceso al blog paratucuidadoenlinea}

El blog paratucuidadoenlinea fue utilizado por los participantes como estrategia para obtener algún tipo de soporte social, indistintamente de variables relacionadas con el 
nivel socioeconómico, grado de escolaridad, ocupación o tipo de enfermedad.

Para el uso de esta herramienta se requiere contar con equipos disponibles a los que el participante puede acceder no solo desde su casa, sino a través de aulas de informática o salas de café internet.

El tiempo de capacitación en el uso de la herramienta depende de aspectos como: conocimientos previos del participante en informática, habilidades en el uso del computador y contar con la posibilidad de realizar ejercicios prácticos diferentes a los que utiliza el orientador. La capacitación se puede brindar de forma presencial o virtual, así mismo se identifica que no es una limitante para ingresar a la herramienta el que el participante no tenga conocimientos previos en informática. Esta variable, como ya se menciono, condicionará el tiempo requerido de entrenamiento.

Las estrategias de interacción propuestas de mayor uso por parte de los participantes son: los temas de consulta expuestos en la pantalla principal del blog con acceso directo, seguido por el foro y el chat; la de menor uso fue la de consultas virtuales a través de correo electrónico.

Posibles limitantes que pueden incidir en el uso de la herramienta son el desconocimiento respecto al acceso y beneficios de la herramienta, incredulidad de los participantes infundada por miembros de la familia y amigos, el temor asociado al uso del computador cuando no se han tenido experiencias previas y, el curso mismo de la enfermedad.

\section{Percepción y significado del uso del blog paratucuidadoenlinea}

El análisis de los casos a partir de la información obtenida en los diarios de campo y las entrevistas semiestructuradas, permite presentar la percepción y el significado del soporte social con uso de Blog. Para una mejor comprensión del proceso se citan los códigos descriptivos y nominales en el Cuadro 1.

En el estudio se encontraron 42 códigos que representaron la esencia de los testimonios de los participantes. La recopilación de los significados formulados fue organizada posteriormente en categorías que emergie-

Cuadro 1. Ejemplo codificación con expresiones de los participantes de la investigación.

\begin{tabular}{|c|c|}
\hline CÓDIGO DESCRIPTIVO & CÓDIGO NOMINAL \\
\hline $\begin{array}{l}\text { "Un saludo muy especial de amor y amistad para todo el grupo de cuidado a cui- } \\
\text { dadores". } \\
\text { "Debemos compartir con las demás personas". }\end{array}$ & Integración con otros \\
\hline $\begin{array}{l}\text { "Hola amigos del grupo estoy feliz de poder participar y sentirme parte de este } \\
\text { grupo". }\end{array}$ & $\begin{array}{l}\text { Sentirse parte de un } \\
\text { grupo }\end{array}$ \\
\hline $\begin{array}{l}\text { "Con respecto a la glucometría a qué hora se debe tomar, es necesario todos los } \\
\text { días? También para comentar que a veces se presentan cambios en la visión quisiera } \\
\text { saber si tiene que ver con el azúcar alto. A veces al aplicar la insulina se presenta una } \\
\text { especie de mareo leve, no puedo ver bien pues es como si estuviera mirando el sol a } \\
\text { través de un espejo pero estos síntomas pasan en unos minutos". }\end{array}$ & $\begin{array}{l}\text { Información para } \\
\text { acciones de cuidado }\end{array}$ \\
\hline $\begin{array}{l}\text { "Deseo estar en comunicación con ustedes, para compartir mi experiencia como } \\
\text { cuidador primario, fue algo especial que Dios me permitió". }\end{array}$ & $\begin{array}{l}\text { Otros medios de } \\
\text { información }\end{array}$ \\
\hline
\end{tabular}




\begin{tabular}{|c|c|}
\hline $\begin{array}{l}\text { "Les quiero contar que me ha ido muy bien con los exámenes para la cirugía... ya } \\
\text { terminé... me falta esperar los resultados haber... falta uno el viernes } 3 \text { de noviem- } \\
\text { bre, ese día también tengo cita con el oncólogo... llevo } 15 \text { días haciéndome esos } \\
\text { exámenes y pues unos me salieron mal y me tocó volverlos a repetir... para parti- } \\
\text { cipar en más en el blog, tengo que esperar qué me dice el médico la otra semana”. } \\
\text { "El tratamiento ha estado bien.. El lunes fui, estuve en la Fundación y me quitaron } \\
\text { el ventiúnico punto que tenía del drenaje y me miraron que súper bien... y ayer en } \\
\text { el hospital de Fontibón y que súper bien... nadita solo con medicamentos... ace- } \\
\text { taminofén, omeprazol, aspirina... y el médico me dijo que me terminara de tomar } \\
\text { todo eso... que en un mes tengo control con él”. }\end{array}$ & Retroalimentación \\
\hline $\begin{array}{l}\text { "Deseo estar en comunicación para compartir mi experiencia como cuidador, Dios } \\
\text { lo permitió como algo especial para mi vida". }\end{array}$ & Compartir la vivencia \\
\hline $\begin{array}{l}\text { "el video me pareció interesante porque nos enseña que debemos ser constantes } \\
\text { para conseguir lo que nos interesa en la vida sobre todo no actuar solos y entender } \\
\text { que se debe buscar ayuda de los profesionales". }\end{array}$ & Aprendizaje \\
\hline $\begin{array}{l}\text { "Aprender a manejar estos programas es algo que me ha llamado la atención. Agra- } \\
\text { dezco muchísimo toda la paciencia y los conocimientos que he podido adquirir" } \\
\text { "practicando el manejo del computador y del internet. He pasado una tarde muy } \\
\text { agradable muchas gracias. Adiós!" }\end{array}$ & Agradecimiento \\
\hline
\end{tabular}

ron del análisis de los códigos nominales.

En el Cuadro 2 se presentan las categorías y códigos que las conformaron. La descripción de cada categoría se contrastó con el texto original con el fin de ser validada, buscando ver si había algo en ese texto que no hubiese sido tenido en cuenta en el grupo de temas y si el grupo de temas proponía algo que no hubiese estado en el texto original.

Por otro lado, se determinó el uso de las estrategias de interacción propuestas de acuerdo a las categorías identificadas:

Cuadro 2: Categorías identificadas en casos de personas con enfermedades crónicas que utilizaron el blog como estrategias de soporte social. Fuente: Investigación junio 2011.

\begin{tabular}{|l|l|}
\hline \multicolumn{1}{|c|}{ CÓDIGOS } & \multicolumn{1}{|c|}{ CATEGORÍA } \\
\hline $\begin{array}{l}\text { Fortalecimiento, Integración con otros, Interacción social, } \\
\text { Potencialidades, Falencias, Sentirse importante, } \\
\text { reconocimiento }\end{array}$ & Autorreconocimiento y crecimiento personal \\
\hline $\begin{array}{l}\text { Denominación, Afecto, Fortalecimiento, Socialización, } \\
\text { Compartir la vivencia, Integración, Interacción social }\end{array}$ & Vínculo \\
Participación en un grupo & Comunicación \\
\hline $\begin{array}{l}\text { Consulta, Asesoría, Acompañamiento, Medio para } \\
\text { informarse en convalecencia, Otros medios de información, } \\
\text { Atención, Uso de medios on-line }\end{array}$ & \\
\hline
\end{tabular}




\begin{tabular}{|l|l|}
\hline $\begin{array}{l}\text { Información oportuna, Aprendizaje, Aplicabilidad, } \\
\text { Descarga emocional, Manejo de medicamentos, }\end{array}$ & Utilidad \\
Respuesta a necesidades, Retroalimentación, & \\
Generar interrogantes, Manejo de emergencias en casa & \\
\hline $\begin{array}{l}\text { Recreación, Esparcimiento, Facilidad de acceso, } \\
\text { agradecimiento, potencialidades, creatividad }\end{array}$ & Satisfacción \\
\hline $\begin{array}{l}\text { Confrontación, acompañamiento, información para } \\
\text { acciones de cuidado, reflexión, adaptación }\end{array}$ & Respuestas
\end{tabular}

Temas de consulta expuestos en la pantalla principal del blog con acceso directo: representó para los participantes utilidad, respuestas, satisfacción y comunicación. En el foro y el chat se identificaron las categorías de respuestas, comunicación, satisfacción y utilidad. Se resalta en esta estrategia la evidencia de las categorías vínculo y auto-reconocimiento y crecimiento personal, lo que permite suponer el alcance e impacto que puede representar este tipo de soporte social, en la medida en que los participantes logran compartir la vivencia, sentirse parte de un grupo, socializar, interactuar, sentirse importante y reconocerse, entre otros aspectos.

Consultas virtuales a través de correo electrónico: se identificaron las categorías de comunicación, satisfacción, respuestas y utilidad. Aunque fue la estrategia que menos utilizaron los participantes, es de resaltar que en las categorías de respuestas y utilidad se reconoce el acompañamiento brindado por el orientador y la consideración de ser un espacio para "descarga emocional".

\section{DISCUSIÓN Y COMENTARIO}

La información que proporcionaron los casos facilito el planteamiento de hipótesis susceptibles de ser evaluadas con mayor exacti- tud en investigaciones posteriores.

Esta investigación refleja la percepción y el significado del soporte social con uso de tecnologías, que expresan las personas con enfermedades crónicas, en la que se determina que sí es posible brindar soporte social con el uso de herramientas tecnológicas como el internet, a través de protocolos de intervención costo-efectivos, centrados en las necesidades de los usuarios, con recurso humano idóneo y con adecuados sistemas de seguimiento, monitoreo y control.

Estos hallazgos para el contexto colombiano no se habían documentado antes y se constituyen en un marco de referencia clave para la movilización de recursos y consolidación de propuestas relacionadas con cuidado en el hogar, planes de egreso hospitalario y modelos de cuidado integral para las personas con enfermedades crónicas utilizando herramientas tecnológicas. El estudio reafirma la relevancia que tiene brindar soporte social a través de grupos formales, a personas con enfermedades crónicas, evidenciándose que la condición de cronicidad genera permanentes necesidades que le implican al receptor del cuidado vincularse a diversas estrategias o grupos de apoyo.

Barrera et al. (2) y Pinto (19), indican que aspectos como el deterioro progresivo, la polifarmacia, la alteración de la funcionalidad, los grados de dependencia, son factores que 
promueven el uso de redes de apoyo social.

Se reconoce que la utilización de herramientas tecnológicas como el internet para brindar soporte social a personas con enfermedades crónicas y cuidadores familiares, es una iniciativa novedosa y pertinente en la generación de propuestas de intervención para el cuidado de la salud. Sin embargo, su aplicación y difusión en el contexto local, con frecuencia está condicionada por percepciones del equipo de salud relacionada con escaso acceso a los recursos tecnológicos por parte de los usuarios y falta de indicadores empíricos verificables que reporten efectividad frente a los esquemas tradicionales de atención.

Sin embargo el análisis del perfil de los casos, determinan que variables como el nivel de escolaridad, estrato socio-económico y ocupación de las personas con enfermedades crónicas no son limitantes para su vinculación al blog ni a otras estrategias de soporte social en línea, sin embargo son factores que se deben analizar en cuanto al recurso humano y material, así como el tiempo requerido para la capacitación y entrenamiento en el uso de la herramienta.

Rogers y Scott (20) en la teoría de las innovaciones hace referencia a que las aplicaciones de ideas, objetos o tecnologías, depende directamente de las características sociales de quien reciba la innovación y sea el encargado de difundirla, entendiendo la difusión como el proceso por medio del cual una innovación se comunica a través de distintos canales en los miembros de un sistema a través del tiempo.

Para que la difusión de la innovación, entendida como una idea, práctica u objeto que es nueva para el individuo y los miembros de su red social se lleve a cabo, Siles (21) indica que se requiere analizar de esta los aspectos positivos (ventajas relativas) que tiene con respecto a otras innovaciones, si se adapta (compatibilidad) a la necesidades y características de la población, cuales son las necesidades de educación o entrenamiento que se necesitan para adoptarla (complejidad), si se puede confiar en los aportes y uso de esta innovación (fiabilidad) y se facilita evaluar los resultados obtenidos con la implementación de la innovación (observabilidad).

En cada uno de los casos se determina, a través de las categorías, las ventajas relativas que representan en los usuarios la inclusión de estrategias tecnológicas para brindar soporte social: interacción social, interacción personal, apoyo emocional a través del establecimiento de un vínculo, auto reconocimiento y crecimiento personal, satisfacción y reconocimiento. Estos aspectos se relacionan con estudios en los que se presentan como indicadores los logros que las personas reconocen, a partir de la intervención brindada: bienestar físico y emocional/mental, aprender a establecer objetivos (12), auto reconocer habilidades, utilizar nueva información (13), fortalecer y/o desarrollar la resolución de problemas (20), crear vínculos con otras personas (22), prepararse en el cuidado (23, 24), mejorar relaciones con el equipo de salud tratante (15), potencializar la autoestima, mayor empoderamiento de la situación, percepción de mayor autoeficacia (25) y auto-aprendizaje para adquirir destrezas que le permitan manejar la enfermedad a largo plazo $(10,26)$.

Otros indicadores de efectividad asociados a la intervención en apoyo social con el uso de TICs tales como la disminución del estrés (27), la impulsividad, la falta de cuidado, la depresión, la soledad y el aislamiento (28), ligados por lo general al impacto que la enfermedad crónica genera para la persona enferma $(24,29,30)$.

El blog es compatible y se adapta a las necesidades y características de los usuarios. Los casos refieren que es de fácil acceso, comprensible y atractivo, indistintamente de su nivel educativo, edad o nivel socioeconómico como ya se menciono.

De acuerdo con la revisión de otros estudios, la herramienta tecnológica que más se ha utilizado para proveer soporte social 
en otros escenarios es el internet $(3,16,23)$ seguido por el teléfono y la combinación del teléfono y el internet (31-33). Respecto al internet, los medios más comunes son el cuestionario en línea, el correo electrónico, el software, la capacitación en línea en informática, las comunidades en línea, las discusiones en línea y el chat, siendo el cuestionario en línea el más utilizado (34), en contraste con lo identificado en el estudio, en donde se identifica que las interacciones propuestas la de mayor uso por parte de los participantes son: los temas de consulta expuestos en la pantalla principal del blog con acceso directo, seguido por el foro y el chat y las consultas virtuales a través de correo electrónico.

Las necesidades de educación o entrenamiento para adoptar la innovación se relacionan con el uso del computador, el acceso a internet y el manejo del blog. Para ello se debe determinar los conocimientos previos de los participantes, los recursos con que cuenta en casa, utilizar ayudas didácticas complementarias (manuales, guías) y disponer de un tiempo aproximado para la capacitación como tal en aulas de informática.

La fiabilidad (confianza en los aportes y uso de esta innovación) de la estrategia esta estrechamente relacionada con las percepciones y el significado que le atribuyen los participantes a la intervención, factores que se constituyen en insumo clave para desarrollar escalas de medición de percepción de soporte social con el uso de las tecnologías en el contexto colombiano, que puedan demostrar con precisión aportes y uso de esta innovación para el cuidado de la salud.

La observabilidad, es decir la facilidad para evaluar los resultados obtenidos con la implementación de la estrategia tecnológica para brindar soporte social, implica establecer un sistema de seguimiento establecido por los orientadores de la intervención, a través de la determinación de indicadores de acceso, uso y apropiación como tal (conta- dor de visitas, frecuencia de ingreso al blog de cada participante, etcétera).

En esta investigación se explora el internet y específicamente el uso del blog como canal de comunicación que permitió transmitir información oportuna, de fácil acceso, comprensible y atractiva para todo tipo de usuarios. Los participantes de la intervención, por medio de una red, a su vez se convirtieron en canal para compartir la información y convocar a más personas en el uso de la herramienta.

Rogers, propone que es fundamental analizar el sistema social en el que se desenvuelve el sujeto que va adoptar la innovación. La adopción, el rechazo y que requiere para este proceso, se ve influenciado por la cultura, las normas, por quien este liderado, la situación económica y muchos otros factores sociales que pueden condicionar o cambiar el curso de la adopción de la innovación.

Es de resaltar, que algunos participantes refirieron temor de "enfrentarse al computador" por las preconcepciones infundadas por integrantes de la familia respecto a informática. Por otro lado, la vivencia como tal de ser persona con enfermedad crónica puede condicionar el uso de la estrategia en cuanto a disponibilidad de tiempo y facilidad de acceso a la herramienta. Estos aspectos permiten concluir que se deben utilizar estrategias tecnológicas complementarias tales como el internet y el teléfono, mantener un sistema de acompañamiento y seguimiento activo y facilitar la comunicación entre los participantes para que compartan la vivencia como tal.

El estudio evidencia que las personas con enfermedades crónicas se interesan por obtener información oportuna relacionada con el manejo de la enfermedad y los medicamentos, aspectos que se relacionan con lo reportado en otras investigaciones en las que los participantes buscan las redes de apoyo social y el internet con el fin de obtener información clara y precisa sobre el diagnósti- 
co y tratamiento de las diferentes patologías (26, 30, 35-39).

Por otro lado se destaca la identificación de las categorías vínculo, auto-reconocimiento y crecimiento personal como variables que lograron obtener los casos dentro del blog, las cuales se generaron gracias a la constitución de la red de apoyo social como tal, y al convencimiento de los casos de estas inmersos dentro de un grupo que les permitía compartir emociones, sentimientos y experiencias del día a día. Como variables que se deben estudiar en otros estudios se identifican las que se asocian a las limitaciones en el uso de la herramienta tales como: desconocimiento, crisis, Incredulidad, múltiples tareas, tratamientos, dificultad en el acceso a recursos y temor; las cuales se relacionan estrechamente con lo referido en la Teoría de Rogers de adopción de innovaciones en las dimensiones que facilitan la difusión de las tecnologías.

Se espera dar continuidad a la investigación con la exploración de cada una de las estrategias utilizadas a través del blog, la inclusión de redes de soporte social en línea y la generación de una escala de soporte social con uso de tecnologías de la comunicación y la información acorde al contexto latinoamericano, colombiano y local.

Así mismo se espera vincular a los nodos de la Red Latinoamericana de Cuidado a la persona con enfermedad crónica y su cuidador familiar, en el que a través de estudios de casos se determine el significado, percepción y aplicabilidad que tienen este tipo de propuestas en diferentes contextos locales y regionales.

Finalmente, es indiscutible la sensibilización y concientización del recurso humano en salud respecto a la inclusión del uso de herramientas tecnológicas como estrategias fundamentales para brindar soporte social y en la optimización de programas de seguimiento y continuidad de las terapéuticas instauradas.

\section{REFERENCIAS}

1. Barrera L, Pinto N, Sánchez B. Evaluación de un programa para fortalecer a los cuidadores familiares de enfermos crónicos. Rev Salud Pública (Bogotá) 2006; 8(2): 14-152.

2. Barrera L, Pinto N, Sánchez B, Carrillo G, Chaparro L. Cuidando a los cuidadores familiares de personas con enfermedad crónica. 1ra ed. Bogotá: Universidad Nacional de Colombia. 2010.

3. Cárdenas DC, Melenge B, Pinilla J, Carrillo GM, Chaparro OL. Soporte social con el uso de las TIC para cuidadores familiares de personas con enfermedad crónica: un estado del arte. Aquichan. 2010; 10(3): 204-213.

4. Nahm ES, Resnick B, Gaines J. Testing the Reliability and Validity of Computer mediated Social Support Measures Among Older Adults A Pilot Study. Comput Inform Nurs. 2004; 22 (4): 211219.

5. Nahm ES, Resnick B. Homebound older adults' experiences with the Internet and Email. Comput Inform Nurs. 2001; 19: 257-263.

6. Wellman B. Computer networks as social networks. Science. 2001; 293:2031-2034.

7. Struk C, Moss J. Focus on Technology: What Can You Do to Move the Vision Forward? Comput Inform Nurs. 2009; 27(3): 193.

8. Rotondi AJ, Sinkule J, Spring M. An Interactive Web-based Intervention for Persons with TBI and Their Families. J Head Trauma Rehabil. 2005; 20(2): 173185.

9. Barrera L, Pinto N, Sánchez B. Red de investigadores en: cuidado a cuidadores de pacientes crónicos. Colombia. Aquichan, 2007; 7(2): 199-206.

10. Klemm P, Wheeler E. Cancer Caregivers Online: Hope, emotional roller coaster, 
and physical/emotional/psychological responses. Comput Inform Nurs. 2005; 23(1): 38-45.

11. Barrera L, Pinto N, Sánchez B. Hacia la construcción de un modelo de cuidado de cuidadores de personas con enfermedad crónica. Actualizaciones en Enfermería. 2008; 11(2): 22-28.

12. Weinert C, Cudney S, Winters C. Social Support in Cyberspace. The next Generation. Comput Inform Nurs. 2005; 23(1): 7-15.

13. Klemm P, Reppert K, Visich L. A nontraditional cancer support group: the Internet. Comput Nurs. 1998;16(1): 31-36.

14. Grant J, Elliot T, Weaver M, Bartolucci A, Newman J. Telephone Intervention with Family Caregivers of Stroke Survivors after Rehabilitation. Stroke. 2002; 33(8): 2060-2065.

15. Shaw BR, Hawkins R, Arora N, McTavish F, Pingree S, Gustafson DH. An exploratory study of predictors of participation in a computer support group for women with Breast cancer. Comput Inform Nurs. 2004; 22(5): 282-288.

16. Eastin MS, Larose R. Alt. support: modeling social support online. Comput Human Behavior. 2005; 21(6): 977-992.

17. Hilbert G. Measuring social support in chronic illness. En Strickland O, Waltz C, editors. Measurement of nursing outcomes. Vol. 4. Measuring clients self care and coping skills. New York: Springer, 1990. p. 79-91.

18. Polit D, Hungler B. Investigación científica en ciencias de la salud. 6ta ed. México: McGraw-Hill Interamericana. 2000; p. 241.

19. Pinto N. Cuidar en el hogar. A personas con enfermedad crónica, su familia y cuidador. 1ra ed. Bogotá: Universidad Nacional de Colombia. 2010.

20. Rogers E, Scott K. The diffusion of Innovations Model and Outreach from the national network of libraries of medicine to native communities. Departament of Communication and Journalism. University of New Mexico. 1997; December 10: 1-12.

21. Siles I. Sobre el uso de las tecnologías en la sociedad tres perspectivas teóricas para el estudio de las tecnologías de la comunicación. Reflexiones. 2004; 83(2): 73-82.

22. Heidrich SM. Mechanisms related to psychological well-being in older women with chronic illnesses: age and disease comparisons. Res Nurs Health. 1996; 19(3): 225-235.

23. Buis L. Emotional and Informational Support Messages in an Online Hospice Support Community. Comput Inform Nurs. 2008; 26(6): 358-367.

24. Hill W, Weinert C. An evaluation of an online intervention to provide social support and health education. Comput Inform Nurs. 2004; 22(5): 282-288.

25. Gallagher D, Gray HL, Tang PC, Chun $\mathrm{Yu} \mathrm{Pu}$, Leung LY, Wang PC, et al. Impact of In-Home Behavioral Management Versus Telephone Support to Reduce Depressive Symptoms and Perceived Stress in Chinese Caregivers: Results of a Pilot Study. Am J Geriatr Psychiatry. 2007; 15(5): 425-434.

26. Pierce L, Steiner V, Govoni AL, Hicks B, Cervantes TL, Friedemann ML. Caregivers Pull Together and Feel Connected in Caring for Persons with Stroke. J Neurosci Nurs. 2004; 36(1): 32-39.

27. Cudney SA, Weinert C. Computer-based support groups. Nursing in cyberspace. Comput Nurs.2000;18(1): 35-43.

28. Weinert C. Social support in cyberspace for women with chronic illness. Rehabil Nurs. 2000; 25(4): 129-135.

29. Sánchez B. La experiencia de ser cuidadora de una persona en situación de enfermedad crónica. Investigación y Educación en Enfermería. 2001; 19(2): 36-51.

30. Mason B, Harrison B. Telephone interventions for family caregivers of patients 
with dementia: what are best nursing practices? Holist Nurs Pract. 2008; 22(6): 348-354.

31. Glueckauf RL, Ketterson TU, Loomis JS, Dages P. Online support and education for dementia caregivers: overview, utilization, and initial program evaluation. Telemed J E Health. 2004; 10(2): 223232.

32. Pierce LL, Steiner V, Govoni AL. In-home online support for caregivers of survivors of stroke: a feasibility study. Comput Inform Nurs. 2002; 20(4): 157-164.

33. White M, Dorman SM. Receiving social support online: implications for health education. Health Educ Res. 2001;16 (6): 693-707.

34. Gustafson D, Hawkins R, Pingree S. Effect of computer support on younger women with breast cancer. J Gen Intern Med. 2001; 16(7): 435-445.

35. Lewis D, Gunawardena S, El Saadawi G. Caring connection developing an inter- net resource for family caregivers of children with cancer. Comput Inform Nurs. 2005; 23(5): 265-274.

36. Eysenbach G, Powell J, Englesakis M, Rizo C, Stern A. Health related virtual communities and electronic support groups: systematic review of the effects of online peer to peer interactions. BMJ. 2004; 328(7449): 1166.

37. Ramos JD, Rai-Chaudhuri A, Neill RW. International online discussion lists on chronic myelogenous leukaemia. BMJ. 2004; 328(7449): 1177-1178.

38. Lester J, Prady S, Finegan Y, Hoch D. Learning from e-patients at Massachusetts General Hospital. BMJ. 2004; 328(7449): 1188-1190.

39. Winkelman WJ, Choo CW. Providersponsored virtual communities for chronic patients: improving health outcomes through organizational patient-centered knowledge management. Health Expect. 2003; 6(4):352-358. 